\title{
Intraoperative Rise of Surface Temperature Monitors Success of Sympathectomy in Palmoaxillary Hyperhidrosis
}

\author{
Sebastian Krämer ${ }^{1}$, Axel Skuballa ${ }^{1}$, Uwe Eichfeld ${ }^{2}$ \\ ${ }^{1}$ Department of Thoracic Surgery, Klinikum St. Georg gGmbH, Leipzig, Germany; ${ }^{2}$ Department of Surgery II, Abdominal, Trans- \\ plant, Thoracic and Vascular Surgery, University Hospital Leipzig, Leipzig, Germany. \\ Email: sebastian.kraemer@sanktgeorg.de
}

Received December $13^{\text {th }}, 2012$; revised January $16^{\text {th }}, 2013$; accepted January $29^{\text {th }}, 2013$

\begin{abstract}
Objectives: Patients with hyperhidrosis suffer from an extreme perspiration that cannot be aligned with natural or situational standards. Endoscopic sympathectomy is a meaningful option for palmar and axillary hyperhidrosis. A standardized method of monitoring the immediate intraoperative success has not been established yet. The presented investigation shows one proposed sollution by monitoring skin surface temperature. The main aspect is to demonstrate a significant rise in temperature with utility for monitoring the immediate success of surgery. Methods: Twenty patients with primary hyperhidrosis were observed and treated in a standardized setting against a control group $(\mathrm{n}=10)$. We obtained diverse data that permit determination of a point of time of measurement of surface temperature and definition of a degree of temperature variance. Results: After 5 minutes a significant change of $0.5^{\circ}$ Celcius was noted on the palms; after 10 minutes on average $1.2^{\circ}$ Celcius. Axillary temperature had significantly changed after 10 minutes with a mean temperature variation of $0.8^{\circ}$ Celcius on the right side and $0.6^{\circ}$ Celcius on the left side. Conclusions: Under consideration of appropriate time intervals of measurement and determined changes in surface temperature an early control of correct clip application in ETS is possible. In the palmar aspect an increase of $0.5^{\circ}$ Celcius at an 5 minutes interval, and more than $1^{\circ}$ Celcius at 10 minutes after placement of the clip as compared to basic values before application of the clip can be proposed.
\end{abstract}

Keywords: Hyperhidrosis; Sympathectomy; Temperature; Monitoring

\section{Objectives}

Sweating is a physiological mechanism of thermo regulation, among other effects. Patients with hyperhidrosis suffer from an extreme perspiration that cannot be aligned with natural or situational standards. Hyperhidrosis is not diagnosed on the basis of a specific quantity of perspiration, but primarily in terms of the patient's medical history [1]. The complex dysregulation of perspiretion results in various degrees of subjective social distress for the patient.

A number of therapeutic procedures are available for the treatment of primary hyperhidrosis. The suitable therapeutic methods should be selected individually and adjusted to the patient's condition and the location of symptoms considering diverse topic therapies, antihidrotic drugs, subcutaneous curettage and the use of botulinum toxin among others. All suitable options of conservative therapy should be exhausted before opting for surgery [2]. Endoscopic thoracic sympathectomy (ETS) is a meaningful option for palmar as well as axillary hyperhidrosis that is resistant to conservative treat- ment.

Performing an ETS in patients with various types of hyperhidrosis is a widely accepted part of the mandatory interdisciplinary and individual treatment concept [3]. The consensus report of 2011 [4] clarified the structured approach. The approaches used for endoscopic thoracoscopic sympathectomy are very different. For ETS treatment of axillary, palmoaxillary and palmoplantoaxillary hyperhidrosis, the consensus report recommends R4 + R5 procedure. The experts expressed no unequivocal decision as regards the technique of intervention. The application of clips can be considered as a practical, rapid, safe, and potentially reversible approach [5-7]. An experienced thoracoscopic surgeon may be able to identify the sympathetic trunk reliably and clearly in terms of its location and level, and thus perform the ETS maneuver successfully. The intervention may be difficult by the appearance of additional neural structures apart from standard nerve pathways, known as so-called alternate neural pathways. As anatomical variations of the sympathetic nervous system, these tender bypass routes play an important role in the success of hyperhidrosis surgery 
[8-10]. To the inexperienced surgeon or the sur- geon faced with ambiguous conditions or structures, a simple and practical low-cost method of intraoperative monitoring would serve as a useful means of controlling the success of ETS. Only estimating the effect of the operative procedure seems to be unacceptable.

In the current study we report on the measurement of surface temperature as a method of intraoperative monitoring of endoscopic thoracoscopic sympathectomy for treatment in palmoaxillary hyperhidrosis. Main issue is to provide a standardized way to presume success. We want to determine a point of time of measurement of surface temperature, to define a degree of temperature variance and to find the best time intervals between measurements.

\section{Methods}

Prospectively twenty patients $(\mathrm{n}=20,11$ men, 9 women. mean age 25.7 years (range $20-35$ )) with therapyrefractory primary axillary hyperhidrosis or palmoaxillary hyperhidrosis were treated and observed in 6-month follow-up (observational time span from January 2010 to June 2011). The investigation was reviewed by the ethics review board of the State Chamber of Physicians of Saxony, Dresden, Germany (IRB approval). We maintained the standard of informed consent. The patients' disease-related medical history was determined preoperatively on the basis of a standardized questionnaire. We used subjective grades of classification ranging from severity grade 1 (absent) to severity grade 6 (maximal conceivable intensity) with reference to degree the feeling of limitation of social contacts in the patient's occupational and personal environment, physical malaise, and optical stress as well as unpleasant odors due to perspiretion. Furthermore, we determined semi-quantitative severity grades from grade 0 to grade 3 according to the guidelines of the AWMF (Arbeits-gemeinschaft der Wissenschaftlichen Medizinischen Fachgesellschaften e.V.Germany's representation of Council for International Organizations of Medical Sciences CIOMS) [11]. Preoperative axillary and palmar perspiration rates (in $\mathrm{mg} / \mathrm{min}$ ) were determined after previous drying of the skin. Using precision scales (KERN EW150 - 3 M, Kern \& Sohn $\mathrm{GmbH}$, Balingen, Germany), we performed gravimetric measurement of the difference in weight on a strip of filter paper before and after 5 minute application of the paper. Surface temperature was measured on both palms and in both armpits using a suitable infrared thermometer (Genius Thermometer, Kendall/COVIDIEN, Mansfield, Massa-chusetts). Both measurements were performed under similar conditions on the second post-operative day. All these parameters should declare and objectify the ETS as successful in treating hyperhidrosis in the described cases.
In the study population eight patients had combined palmoaxillary hyperhidrosis while 12 patients had isolated axillary hyperhidrosis. Secondary hyperhidrosis had been ruled out in all patients. The mean duration of disease was 7 years (range 2 - 15 years). A familiar predisposition was noted in $65 \%$. All patients had used various options of conservative therapy. No patient had undergone ETS previously. All 20 patients underwent the following procedure: single-lung ventilation in general anesthesia in a standardized surgical setting (the temperature setting for the operating room was $22^{\circ} \mathrm{C}$ ), and a bilateral ETS (starting on the right side) in lateral position by means of clipping. No infusion was prewarmed nor applied via iv-access at the patients hands, but at the antecubital fossa area. The type of anesthesia (balanced anesthesia versus total intravenous aneasthesia (TIVA)) was not determined by the setting, but was entirely subject to the anesthetist's discretion. The investigations performed by Kwak et al. support this decision in that body temperature did not differ when using the two types of anesthesia mentioned above [12]. Although Propofol has been reported to cause a lower body temperature in comparison to narcotic gases they were comparable in conditions of mild perioperative hypothermia [13].

After performing three incisions for $5 \mathrm{~mm}$ trocars on the lateral chest wall the sympathetic trunk was exposed under camera guidance $\left(30^{\circ}\right.$ optic, $\left.5 \mathrm{~mm}\right)$ at the level of the 4th and 5th rib using diathermy hooks. At the upper margin of the 4th (topR4) and 5th rib (topR5), one titanium clip each was placed on the sympathetic trunk. In this study we use the 2011 concensus proposed nomenclatur according to the rib level and give up the ganglion related nomenclatur. All patients in our population were treated with topR4 + topR5 clipping, although this approach had not been validated by a consensus decision at the time. Furthermore, linear coagulation was performed during every intervention along the posterior circumference of the 4th rib in order to register any anatomical variations of the sympathetic trunk. Intraoperatively, the core temperature of the body was measured through a nasopharyngeal probe while surface temperature was discontinuously measured on a marked spot on the palm and the axilla immediately before clip application and 1 minute, 5 minutes and 10 minutes after placement of the clip. Following removal of all instruments and trocars, a chest tube (Charrière 18) was placed through the trocar used for the camera. The patient was repositioned. The subsequent procedure was the same as that performed on the contralateral side. The patient was discharged on the third postoperative day.

Further reevaluations were performed after four weeks and six months postoperatively, using a standardized list of questions - either by patients visit or via mail.

To ensure the statistical comparability of intraopera- 
tive data, subsequently we performed a measurement in a control group of patients $(\mathrm{n}=10,6$ men, 4 women, mean age 44.9 years (range 19 - 76 years)) with exclusion of hyperhidrosis who had undergone a video-assisted thoracoscopy with diagnostic partial lung resection using endoscopic surgical stapler. Outer conditions, places and time points of measurement and perioperative aspects were the same to the patients group.

Statistical analysis was performed by using the SPSS statistics (v20.0) program (IBM Deutschland $\mathrm{GmbH}$, Ehningen, Germany). Statistical consultation was provided by the Institute of Medical Statistics and Epidemiology (IMISE) at the University of Leipzig and by the Clinical Trial Center Leipzig, KKSL. Statistical analysis were performed by using Wilcoxon and Mann-Whitney-u-test (confidence intervall 95\%). p-values $<0.05$ were considered to denote statistical significance.

\section{Results}

The mean duration of the hospital stay was 3.05 days. No perioperative complications occurred in this population during this period. Furthermore, all patients $(n=20)$ could be followed up with a list of questions after 2 days, 19 patients after 4 weeks, and 18 patients after 6 months. All patients demonstrated improvement in the symptoms of the disease immediately after surgery as well as during the subsequent course of their recovery. The subjective classification of severity before an after surgery vary clearly (Table 1). Variations in the severity grades according to the AWMF guideline are shown in Table 1. Pre- and post-operative temperatures differ distinctly in the palmar and the axillary aspect as well as for the right the left side (p-values according to Wilcoxon, Table 2). Gravimetric measurements with differences in all four positions ( $\mathrm{p}$-values according to Wilcoxon) are presented

Table 1. Subjective classification of severity and semiobjective classification (AWMF guideline) in follow-up in mean (p-values according to WILCOXON, in relation to preoperative classification).

\begin{tabular}{ccccc}
\hline Subjective classification $(1-6)$ in mean & Preoperative & 2nd postoperative day & 4 weeks postoperative & 6 months postoperative \\
\hline Limitation in social life private & $4.25 \pm 1.3$ & $1.1 \pm 0.3(\mathrm{p}<0.000)$ & $1.11 \pm 0.3(\mathrm{p}<0.000)$ & $1.11 \pm 0.3(\mathrm{p}<0.000)$ \\
Limitation in social life-occupational & $5.4 \pm 0.8$ & $1.3 \pm 0.5(\mathrm{p}<0.000)$ & $1.32 \pm 0.3(\mathrm{p}<0.000)$ & $1.17 \pm 0.3(\mathrm{p}<0.000)$ \\
Physical malaise & $4.95 \pm 0.8$ & $1.2 \pm 0.4(\mathrm{p}<0.000)$ & $1.26 \pm 0.4(\mathrm{p}<0.000)$ & $1.17 \pm 0.3(\mathrm{p}<0.000)$ \\
Optical limitations & $3.05 \pm 1.4$ & $1.1 \pm 0.2(\mathrm{p}<0.000)$ & $1.16 \pm 0.3(\mathrm{p}<0.000)$ & $1.06 \pm 0.2(\mathrm{p}<0.000)$ \\
Unpleasant odors & $4.75 \pm 1.1$ & $1.05 \pm 0.3(\mathrm{p}<0.000)$ & $1.21 \pm 0.3(\mathrm{p}<0.000)$ & $1.06 \pm 0.2(\mathrm{p}<0.000)$ \\
\hline Semiobjective classification AWMF $(1-3)$ & Preoperative & 2 nd postoperative day & 4 weeks postoperative & 6 months postoperative \\
\hline Palms (n) & 8 & 3 & 3 & 2 \\
Severity grade & $1.5 \pm 0.5$ & $1 \pm 0.5$ & $1 \pm 0.5$ & $1 \pm 0.5$ \\
Armpits (n) & 20 & 5 & 6 & 4 \\
Severity grade & $1.95 \pm 0.9$ & $1 \pm 0.4$ & $1 \pm 0.2$ & $1 \pm 0.3$ \\
\hline
\end{tabular}

Table 2. Comparison of pre- and postoperative temperature and gravimetric measurements (p-values according to WILCOXON).

\begin{tabular}{|c|c|c|c|}
\hline & \multicolumn{2}{|c|}{ Temperature in mean } & \multirow[t]{2}{*}{ Mean difference } \\
\hline & Preoperative & 2nd postoperative day & \\
\hline & \multicolumn{3}{|c|}{ Degree Celcius } \\
\hline Palm-right & $30.3( \pm 1.9)$ & $33.2( \pm 1.78)$ & $+2.9( \pm 1.59)(\mathrm{p}<0.000)$ \\
\hline Palm-left & $30.1( \pm 2.1)$ & $33.1( \pm 1.89)$ & $+3.0( \pm 1.66)(\mathrm{p}<0.000)$ \\
\hline Armpit-right & $32.2( \pm 2.0)$ & $34.4( \pm 1.75)$ & $+2.2( \pm 1.28)(\mathrm{p}<0.000)$ \\
\hline Armpit-left & $32.5( \pm 1.9)$ & $34.3( \pm 1.78)$ & $+1.9( \pm 1.59)(\mathrm{p}<0.000)$ \\
\hline \multirow[t]{4}{*}{ Core } & $36.1( \pm 0.9)$ & $35.8( \pm 1.21)$ & $-0.3( \pm 1.08)(\mathrm{p}=0.281)$ \\
\hline & \multicolumn{2}{|c|}{ Gravimetry in mean } & Mean difference \\
\hline & Preoperative & 2nd postoperative day & \\
\hline & \multicolumn{3}{|c|}{$\mathrm{mg} / \mathrm{min}$} \\
\hline Palm-left & $63.3( \pm 138.3)$ & $7.9( \pm 5.8)$ & $55.5( \pm 138.4)(\mathrm{p}<0.000)$ \\
\hline Armpit-right & $123.9( \pm 61.2)$ & $19.8( \pm 13.0)$ & $104.2( \pm 56.8)(\mathrm{p}<0.000)$ \\
\hline Armpit-left & $137.3( \pm 126.8)$ & $19.0( \pm 11.3)$ & $118.3( \pm 128.6)(\mathrm{p}<0.000)$ \\
\hline
\end{tabular}


in Table 2. Compensatory sweating was observed by questionnaire in 4 patients $(20 \%)$ on the second postoperative day, in 4 patients $(21.1 \%)$ after four weeks, and in 6 patients $(33.3 \%)$ after six months.

Intraoperative measurements in patients $(n=20)$ compared to measurements in the control group $(\mathrm{n}=10)$ led to various conclusions presented in Table 3 and shown in Figures 1-3. Body temperature differed in all patients, but there was no evidence of significance (Mann-Whitney U-test). The temperature in the operating
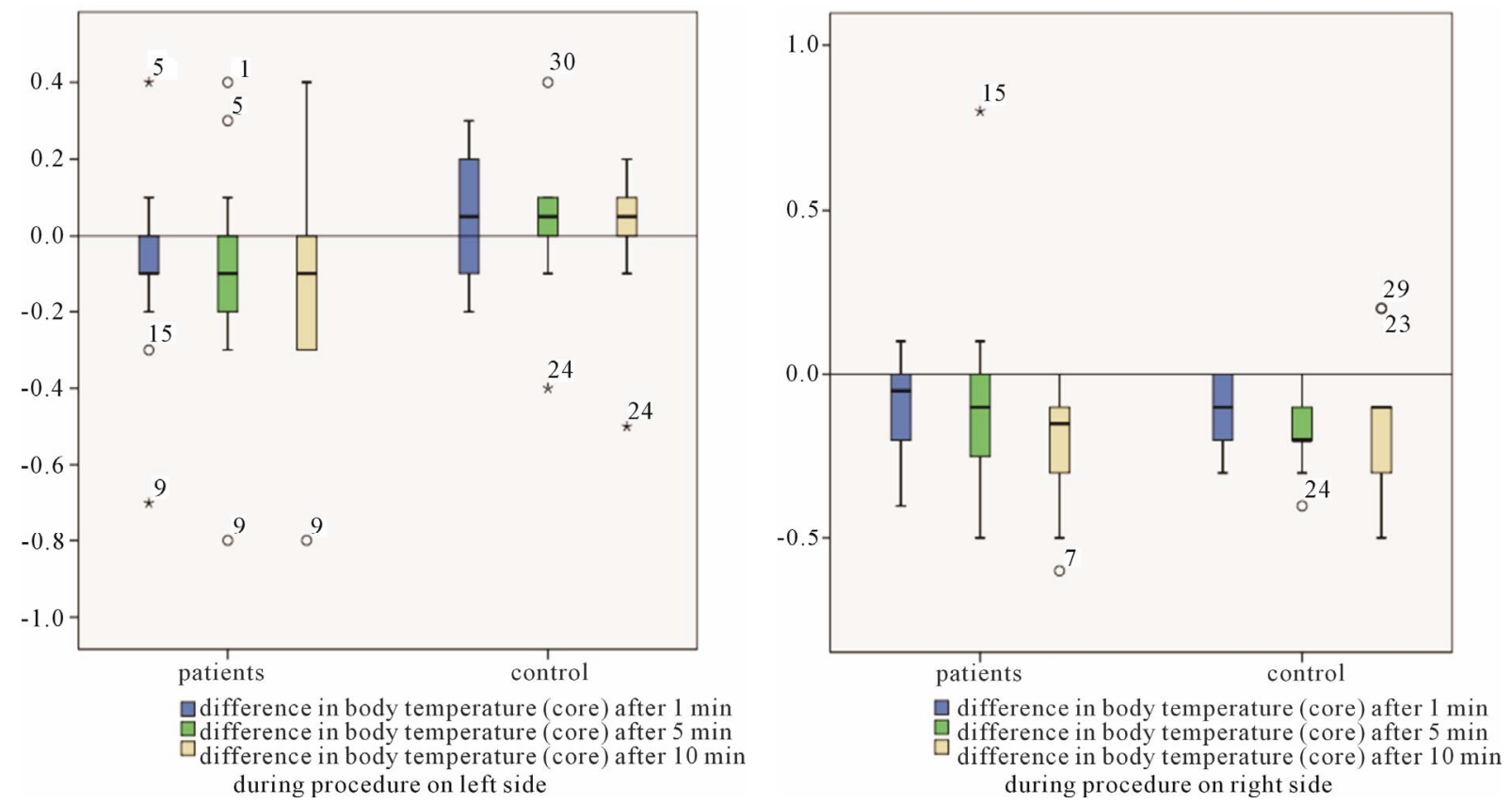

Figure 1. Comparison of intraoperative measurements between patients group and controll group-difference in body temperature (core).

Table 3. Intraoperative measurements in patients compared to measurements in the control group in mean (p-value according to Mann-Whitney U-test), body temperature data collected separately for corresponding side.

\begin{tabular}{|c|c|c|c|c|c|c|}
\hline \multirow{2}{*}{ Body core temperature } & \multicolumn{2}{|c|}{ Patients } & \multicolumn{2}{|c|}{ Control } & \multicolumn{2}{|c|}{$\mathrm{p}$-value } \\
\hline & right & left & right & left & right & left \\
\hline \multicolumn{7}{|c|}{ Degree Celcius } \\
\hline DeltaT after $1 \mathrm{~min}$ & $-0.1 \pm 0.13$ & $-0.1 \pm 0.20$ & $-0.1 \pm 0.10$ & $0 \pm 0.17$ & 0.508 & 0.06 \\
\hline DeltaT after $5 \mathrm{~min}$ & $-0.1 \pm 0.27$ & $-0.1 \pm 0.25$ & $-0.2 \pm 0.13$ & $0 \pm 0.20$ & 0.671 & 0.136 \\
\hline DeltaT after $10 \mathrm{~min}$ & $-0.3 \pm 0.17$ & $-0.1 \pm 0.25$ & $-0.1 \pm 0.21$ & $0 \pm 0.19$ & 0.587 & 0.089 \\
\hline \multirow{2}{*}{ Temperature armpits } & \multicolumn{2}{|c|}{ Patients } & \multicolumn{2}{|c|}{ Control } & \multicolumn{2}{|c|}{$\mathrm{p}$-value } \\
\hline & right & left & right & left & right & left \\
\hline \multicolumn{7}{|c|}{ Degree Celcius } \\
\hline DeltaT after $1 \mathrm{~min}$ & $-0.1 \pm 0.78$ & $-0.1 \pm 0.66$ & $0 \pm 0.21$ & $0 \pm 0.21$ & 0.809 & 0.758 \\
\hline DeltaT after $5 \mathrm{~min}$ & $0.1 \pm 0.78$ & $0.2 \pm 0.73$ & $0 \pm 0.16$ & $0 \pm 0.19$ & 0.165 & 0.186 \\
\hline Delta $T$ after $10 \mathrm{~min}$ & $0.8 \pm 0.76$ & $0.6 \pm 0.87$ & $-0.1 \pm 0.16$ & $0 \pm 0.23$ & $<0.000$ & 0.015 \\
\hline \multirow{2}{*}{ Temperature palms } & \multicolumn{2}{|c|}{ Patients } & \multicolumn{2}{|c|}{ Control } & \multicolumn{2}{|c|}{ p-value } \\
\hline & right & left & right & left & right & left \\
\hline \multicolumn{7}{|c|}{ Degree Celcius } \\
\hline DeltaT after $1 \mathrm{~min}$ & $-0.4 \pm 0.61$ & $-0.2 \pm 0.57$ & $-0.1 \pm 0.17$ & $0 \pm 0.08$ & 0.1158 & 0.268 \\
\hline DeltaT after $5 \mathrm{~min}$ & $0.5 \pm 1.02$ & $0.5 \pm 1.13$ & $-0.2 \pm 0.25$ & $-0.2 \pm 0.15$ & 0.011 & 0.009 \\
\hline DeltaT after $10 \mathrm{~min}$ & $1.2 \pm 0.81$ & $1.3 \pm 1.02$ & $-0.1 \pm 0.24$ & $-.2 \pm 0.21$ & $<0.000$ & $<0.000$ \\
\hline
\end{tabular}



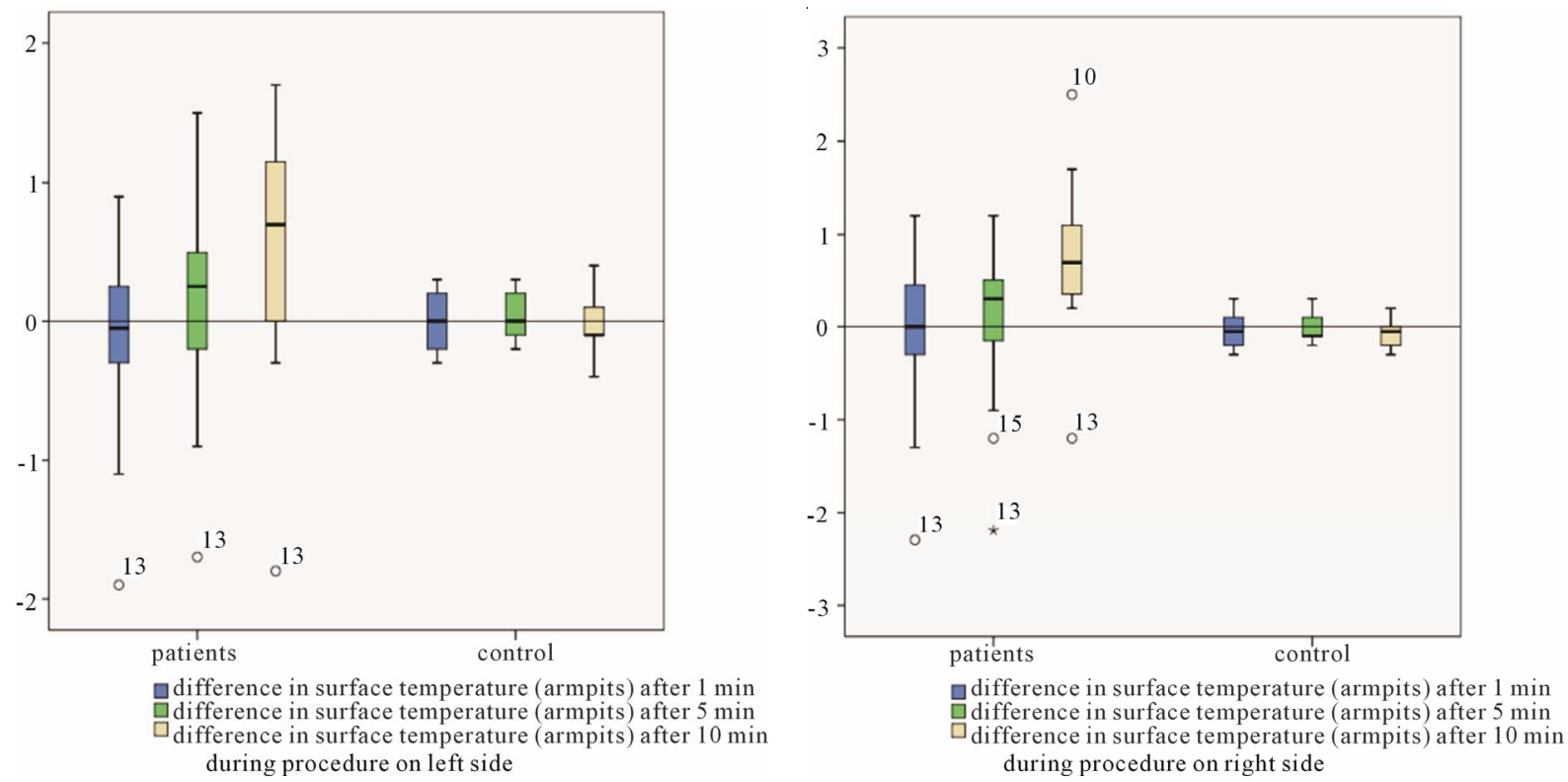

Figure 2. Comparison of intraoperative measurements between patients group and controll group-difference in surface temperature (armpits).
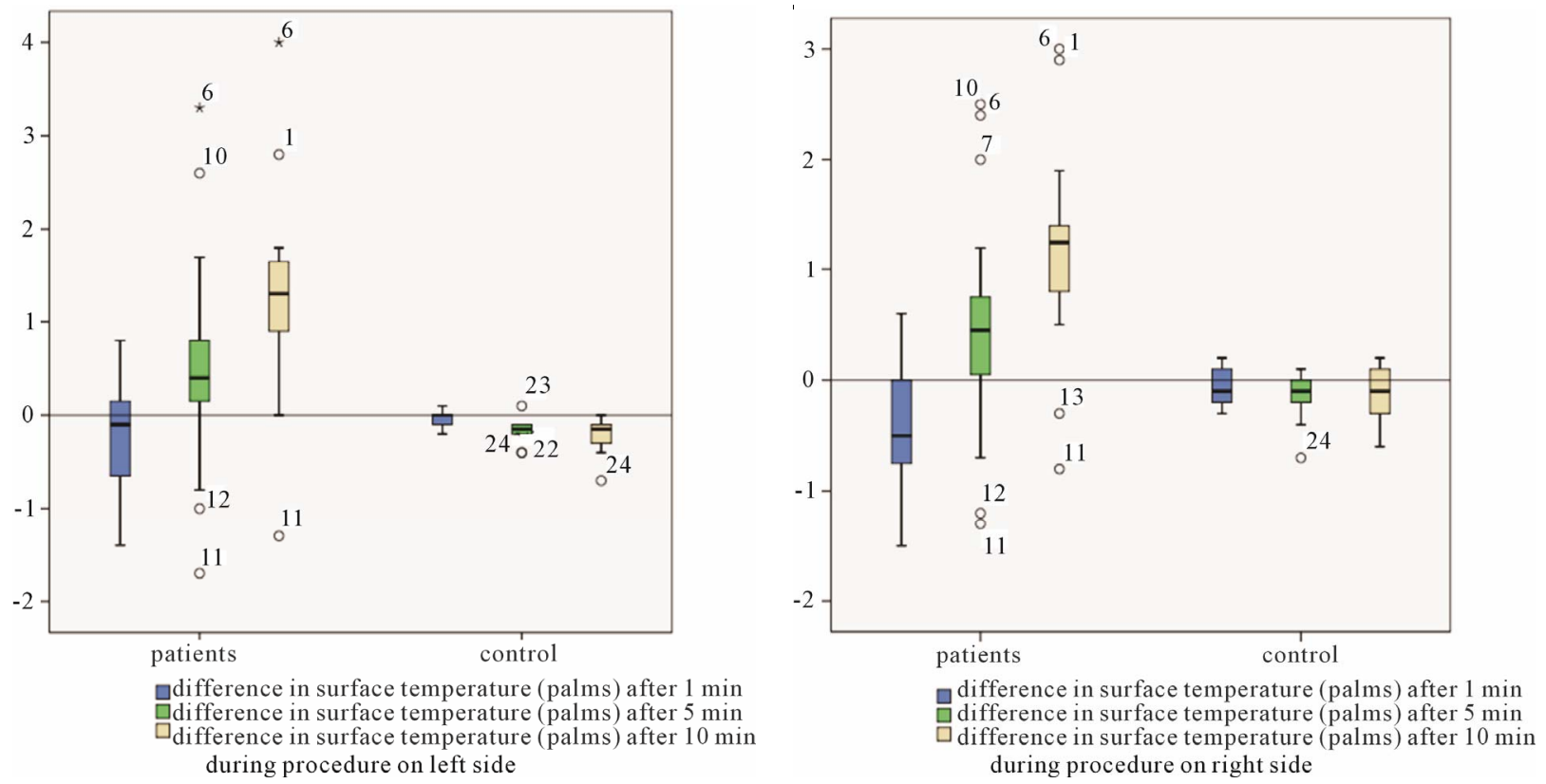

Figure 3. Comparison of intraoperative measurements between patients group and controll group-difference in surface temperature (palms).

room was set to $22^{\circ} \mathrm{C}$ by the central air-conditioning system. In fact the temperature in the operating room was on average $22.2^{\circ} \mathrm{C}\left( \pm 0.5^{\circ}\right.$ Celcius $)$.

\section{Conclusions}

Comparisons of pre- and postoperative measurements of palmar and axillary surface temperature as well as gravimetric measurement of the quantity of perspiration permitted limited objective assessment of the success of treating this condition, which is associated with a very marked degree of subjective distress for the patient. However, these values do not permit objective assessment of the severity of the disease. The AWMF classifications are better established; they are regarded as a semi-objective measurement of the severity of this condition. Graded subjective assessments of the level of distress in respect of various aspects of daily life showed improvement in the patients' quality of life during follow-up. 
Sympathetic denervation causes vasodilatation in the area being supplied by the nerves, thus raising the temperature in the area. Intraoperative measurement of surface temperature is a described method to assess the success of the procedure. Diverse ways of handling can be found $[14,15]$. Time intervalls for measurements and proposed temperature rises at a certain time point are inconsistent or rarely recorded. A continuous skin temperature measurement seems to be more replicable but may be altered by other operative/perioperative steps [16-18]. Monitoring palmar laser Doppler blood flow changes is another meanful option with a better temporal resolution. The cautery of the parietal pleura may lead to a variation before disruption the sympathetic chain. The utility of skin blood-flow measurements in identifying the success of the procedure is interfered by that [19].

We believe it is important to measure the temperture at determined points of time and to note according temperature variances. The data in the present investi- gation allows us to determine an increase of palmar surface temperature of at least $0.5^{\circ}$ Celcius at a 5 minutes interval, and more than $1^{\circ}$ Celcius at 10 minutes after placement of the clip as compared to basic values before application of the clip. The axillary measurements only show an significant increase of more than $0.5^{\circ}$ Celcius at the 10 minutes interval after clip placement, obviously due to the more central body position. It can be concluded that monitoring surface temperature in the palmar aspect is more easily achieved by the cooperation of anesthetists. Under consideration of appropriate time intervals of measurement and determined changes in surface temperature an early control of correct clip application in ETS is possible. It might be useful for the beginner in ETS surgery as well as for the experienced surgeon confronted with ambiguous intraoperative or anatomical conditions. Besides it is in addition to in-situ fotography a further way of documentation.

This technique could have some value if the sympathectomy should be done at the lowest possible level to minimize compensatory sweating with a way to judge success [20]. By now the presented method and the given values are pratical suggestions in the surgical setting in treating palmoaxillary hyperhidrosis.

\section{REFERENCES}

[1] H. W. Walling, "Clinical Differentiation of Primary from Secondary Hyperhidrosis," Journal of the American Academy of Dermatology, Vol. 64, No. 4, 2011, pp. 690-695. doi:10.1016/j.jaad.2010.03.013

[2] R. Reisfeld, K. I. Berliner, "Evidence-Based Review of the Nonsurgical Management of Hyperhidrosis," Thoracic Surgery Clinics, Vol. 18, No. 2, 2008, pp. 157-166. doi:10.1016/j.thorsurg.2008.01.004

[3] T. Schlereth, M. Dieterich and F. Birklein, "Hyperhi-
drosis-Causes and Treatment of Enhanced Sweating," Deutsches Aerzteblatt International, Vol. 106, No. 3, 2009, pp. 32-37.

[4] R. J. Cerfolio, J. R. De Campos, A. S. Bryant, et al., "The Society of Thoracic Surgeons Expert Consensus for the Surgical Treatment of Hyperhidrosis," The Annals of Thoracic Surgery, Vol. 91, No. 5, 2011, pp. 1642-1648. doi:10.1016/j.athoracsur.2011.01.105

[5] H. J. Henteleff and D. Kalavrouziotis, "Evidence-Based Review of the Surgical Management of Hyperhidrosis," Thoracic Surgery Clinics, Vol. 18, No. 2, 2008, pp. 209216. doi:10.1016/j.thorsurg.2008.01.008

[6] C. C. Lin, L. R. Mo, L. S. Lee, S. M. Ng and M. H. Hwang, "Thoracoscopic T2-Sympathetic Block by Clipping-A Better and Reversible Operation for Treatment of Hyperhidrosis Palmaris: Experience with 326 Cases," The European Journal of Surgery, No. 580, 1998, pp. 13-16.

[7] H. Sugimura, E. H. Spratt, C. G. Compeau, D. Kattail and Y. Shargall, "Thoracoscopic Sympathetic Clipping for Hyperhidrosis: Long-Term Results and Reversibility," The Journal of Thoracic and Cardiovascular Surgery, Vol. 137, No. 6, 2009, pp. 1370-1376. doi:10.1016/j.jtcvs.2009.01.008

[8] B. Zhang, Z. Li, X. Yang, et al., "Anatomical Variations of the Upper Thoracic Sympathetic Chain," Clinical Anatomy, Vol. 22, No. 5, 2009, pp. 595-600. doi: $10.1002 /$ ca.20803

[9] L. Ramsaroop, B. Singh, J. Moodley, P. Partab and K. S. Satyapal, "Anatomical Basis for a Successful Upper Limb Sympathectomy in the Thoracoscopic Era," Clinical Anatomy, Vol. 17, No. 4, 2004, pp. 294-299. doi: $10.1002 /$ ca. 10238

[10] I. H. Chung, C. S. Oh, K. S. Koh, H. J. Kim, H. C. Paik and D. Y. Lee, "Anatomic Variations of the T2 Nerve Root (Including the Nerve of Kuntz) and Their Implications for Sympathectomy," The Journal of Thoracic and Cardiovascular Surgery, Vol. 123, No. 3, 2002, pp. 498-501. doi: $10.1067 / \mathrm{mtc} .2002 .119340$

[11] AWMF-Register Nr. 013/059, Klasse: S1, Definition und Therapie der Primären Hyperhidrose (Valid until 14.01. 2017). http://www.awmf.org

[12] H. J. Kwak, S. K. Min, I. K. Yi, Y. J. Chang and J. Y. Kim, "Comparison of the Effects of Sevoflurane and Propofol on Core Body Temperature during Laparoscopic Abdo- minal Surgery," Korean Journal of Anesthesiology, Vol. 61, No. 2, 2011, pp. 133-137. doi:10.4097/kjae.2011.61.2.133

[13] T. Chowdhury, H. Prabhakar, S. J. Bharati, K. Goyal, S. K. Dube and G. P. Singh, "Comparison of Propofol versus Sevoflurane on Thermoregulation in Patients Undergoing Transsphenoidal Pituitary Surgery: A Preliminary Study," Saudi Journal of Anaesthesia, Vol. 6, No. 1, 2012, pp. 12-15. doi:10.4103/1658-354X.93046

[14] X. Li, Y. R. Tu, M. Lin, F. C. Lai, J. F. Chen and H. W. Miao, "Minimizing Endoscopic Thoracic Sympathectomy for Primary Palmar Hyperhidrosis: Guided by Palmar Skin Temperature and Laser Doppler Blood Flow," The Annals of Thoracic Surgery, Vol. 87, No. 2, 2009, pp. 427-431. 
doi:10.1016/j.athoracsur.2008.10.009

[15] T. Y. Chuang, Y. S. Yen, J. W. Chiu, R. C. Chan, S. C. Chiang, M. P. Hsiao and L. S. Lee, "Intraoperative Monitoring of Skin Temperature Changes of Hands before, during and after Endoscopic Thoracic Sympathectomy: Using Infrared Thermograph and Thermometer for Measurement," Archives of Physical Medicine and Rehabilitation, Vol. 78, No. 1, 1997, pp. 85-88. doi:10.1016/S0003-9993(97)90016-2

[16] J. H. Eisenach, T. L. Pike, D. E. Wick, et al., "A Comparison of Peripheral Skin Blood Flow and Temperature during Endoscopic Thoracic Sympathotomy," Anesthesia \& Analgesia, Vol. 100, No. 1, 2005, pp. 269-276. doi:10.1213/01.ANE.0000139934.15659.7F

[17] I. Ng and T. T. Yeo, "Palmar Hyperhidrosis: Intraoperative Monitoring with Laser Doppler Blood Flow as a Guide for Success after Endoscopic Thoracic Sympathec- tomy," Neurosurgery, Vol. 52, No. 1, 2003, pp. 127-130.

[18] K. Lu, C. L. Liang, T. C. Lee, H. J. Chen, T. M. Su and P. C. Liliang, "Changes of Bilateral Palmar Skin Temperature in Transthoracic Endoscopic T-2 Sympathectomy," Journal of Neurosurgery, Vol. 92, No. S1, 2000, pp. 44-49.

[19] C. G. Crandall, D. M. Meyer, S. L. Davis and S. M. Dellaria, "Palmar Skin Blood Flow and Temperature Responses throughout Endoscopic Sympathectomy," Anesthesia \& Analgesia, Vol. 100, No. 1, 2005, pp. 277-283. doi:10.1213/01.ANE.0000139359.46635.2D

[20] D. L. Miller, A. S. Bryant, S. D. Force and J. I. Miller Jr., "Effect of Sympathectomy Level on the Incidence of Compensatory Hyperhidrosis after Sympathectomy for Palmar Hyperhidrosis," The Journal of Thoracic and Cardiovascular Surgery, Vol. 138, No. 3, 2009, pp. 581-585. doi:10.1016/j.jtcvs.2009.03.059 